\title{
Adrenaline use is associated with excess organ injury and mortality in cardiogenic shock: facts and fiction
}

\author{
Shou-Yin Jiang ${ }^{1}$, Ye-Hua Shen ${ }^{2}$ and Xiao-Gang Zhao ${ }^{{ }^{*}}$ \\ See related research by Tarvasmäki et al. http://ccforum.biomedcentral.com/articles/10.1186/s13054-016-1387-1
}

We read with great interest the article by Tarvasmäki et al. [1], who observed the relationship between vasopressor/inotrope use and outcome in patients with cardiac shock (CS). Their results indicate that use of adrenaline in CS is associated with increased 90-day mortality and marked worsening of cardiac and renal biomarkers during the first few days. The study underscores the need for randomized controlled trials of adrenaline versus noradrenaline in CS.

However, we have to raise a serious question regarding the undetailed subgroup of patients with cardiac arrest (CA) because we consider that those CA patients should have been excluded from this study or at least should be analyzed in the form of a subgroup. According to their results, $39 \%$ of patients received adrenaline prior to inclusion because of CA. After inclusion, $21 \%$ of patients received adrenaline. We doubt whether the "21\%" were mainly those who suffered from CA. This is of vital importance because it may cause an obvious bias. This is true when we review their data. Their Table 1 explicitly indicates that patients treated with adrenaline had more severe complications with higher proportions of confusion, oliguria, and hyperlactacidemia. However, a large number of CA patients can also present with confusion, oliguria, and hyperlactacidemia if they experience a prolonged lowflow or no-flow state. Therefore, it should be realized that the CA patients were inappropriately included.

Adrenaline use is associated with higher oxygen requirements of the heart [2]. The very recent European and American guidelines for the diagnosis and treatment of acute heart failure do not recommend routine use of adrenaline in CS unless patients have persistent hypotension despite adequate cardiac filling pressures and the use of other vasoactive agents and resuscitation $[3,4]$. The use of noradrenaline has been prevalent in the management of critically ill patients and has been given high grade recommendations in guidelines. Thus, we do not think further clinical trials are required to compare adrenaline and noradrenaline for the treatment of CS.

\section{Authors' response}

Tuukka Tarvasmäki, Johan Lassus, Alexandre Mebazaa and Veli-Pekka Harjola

We thank Zhao and colleagues for their interest in our paper. We agree that showing the results of survival analyses excluding resuscitated patients may be of additional value. Hence, in the subgroup of patients that were not resuscitated, the adjusted 90-day mortality odds ratio for adrenaline (versus other vasopressors) was 6.5 (95 \% confidence interval 1.5-27.2, $p=0.01$ ). Figure S2 (Additional

\footnotetext{
* Correspondence: zhaoxiaogang1969@gmail.com

${ }^{1}$ Department of Emergency Medicine, Second Affiliated Hospital, Zhejiang University School of Medicine; Research Institute of Emergency Medicine, Zhejiang University, 88 Jiefang Road, Hangzhou 310009, China

Full list of author information is available at the end of the article
}

file 5) in our original paper [1] confirms the detrimental evolution of cardiac and renal biomarkers in patients treated with adrenaline versus other vasopressors in the subgroup of non-resuscitated patients.

While subgroup analyses in the non-resuscitated patients consistently showed that adrenaline was associated with detrimental effects on outcome, we prefer to present the results for the whole cohort-not specifically excluding patients who had cardiac arrest-as resuscitated patients are part of our daily practice. Indeed, a significant 
proportion of patients ( $28 \%$ in the CardShock study) with cardiogenic shock are resuscitated.

We would also take the opportunity to clarify the numbers regarding the frequency of adrenaline use. Of all patients included in the study, $21 \%(46 / 216)$ were treated with adrenaline, defined as the use of continuous infusion (not bolus injection related to, e.g., resuscitation). However, less than half of the patients treated with continuous adrenaline, $39 \%$ (18/46), were resuscitated prior to study inclusion. On the other hand, $70 \%$ of resuscitated patients were not treated later with continuous adrenaline.

It is true that confusion, oliguria, and elevated lactate levels are very common after cardiac arrest, reflecting inadequate organ perfusion also in this scenario. Nevertheless, signs of hypoperfusion are clinical landmark signs of cardiogenic shock, with or without prior resuscitation.

The recently published European guidelines [3] recommend rather restrictive use of adrenaline in the setting of cardiogenic shock, but the level of evidence is low. The latter might explain the surprisingly common use of adrenaline. Moreover, there are no large randomized studies supporting the recommendation to prefer noradrenaline over adrenaline in CS. Altogether, we still believe that there is room for a pivotal trial to identify the most efficient drug regimen with a favorable safety profile in CS.

\section{Abbreviations}

CA: Cardiac arrest; CS: Cardiac shock; CS: Cardiogenic shock

Availability of supporting data

All data supporting our findings is available through PubMed.

\section{Authors' contributions}

SYJ wrote the letter. YHS and XGZ proofread and edited the letter. All authors have read and approved the final version.

\section{Competing interests}

The authors declare that they have no competing interests.

\section{Author details}

1Department of Emergency Medicine, Second Affiliated Hospital, Zhejiang University School of Medicine; Research Institute of Emergency Medicine, Zhejiang University, 88 Jiefang Road, Hangzhou 310009, China. ${ }^{2}$ Department of Radiology, The Children's Hospital, Zhejiang University School of Medicine, Hangzhou 310009, China.

Published online: 28 September 2016

\section{References}

1. Tarvasmäki T, Lassus J, Varpula M, Sionis A, Sund R, Køber L, et al. Current real-life use of vasopressors and inotropes in cardiogenic shock-adrenaline use is associated with excess organ injury and mortality. Crit Care. 2016;20:1.

2. Krenn L, Delle KG. Essential lessons in cardiogenic shock: epinephrine versus norepinephrine/dobutamine. Crit Care Med. 2011;39:583-4.

3. Ponikowski P, Voors AA, Anker SD, Bueno H, Cleland JG, Coats AJ, et al. 2016 ESC Guidelines for the diagnosis and treatment of acute and chronic heart failure: The Task Force for the diagnosis and treatment of acute and chronic heart failure of the European Society of Cardiology (ESC). Developed with the special contribution of the Heart Failure Association (HFA) of the ESC. Eur J Heart Fail. 2016. doi:10.1002/ejhf.592.
Yancy CW, Jessup M, Bozkurt B, Butler J, Casey Jr DE, Colvin MM, et al. 2016 ACC/AHA/HFSA Focused Update on New Pharmacological Therapy for Heart Failure: An Update of the 2013 ACCF/AHA Guideline for the Management of Heart Failure: A Report of the American College of Cardiology/American Heart Association Task Force on Clinical Practice Guidelines and the Heart Failure Society of America. J Am Coll Cardiol. 2016. in press. 